$\mathrm{J}$ o u r n a l of

Mathematics

and Applications

JMA No 43, pp 5-17 (2020)

\title{
On the Alternative Structures for a Three-Grade Markov Manpower System
}

\author{
Vincent A. Amenaghawon, Virtue U. Ekhosuehi \\ and Augustine A. Osagiede
}

ABSTRACT: This paper considers a manpower system modelled within the Markov chain context under the condition that recruitment is done to replace outgoing flows. The paper takes up the embeddability problem in a three-grade manpower system and examines it from the standpoint of generating function (i.e., the z-transform of stochastic matrices). The method constructs a stochastic matrix that is made up of a limiting-state probability matrix and a partial sum of transient matrices. Examples are provided to illustrate the utility of the method.

AMS Subject Classification: 15A18, 91D35.

Keywords and Phrases: Embeddability problem; Manpower system; Markov chain; Stochastic matrix; Z-transform.

\section{Introduction}

Mathematical models are often used to describe how changes take place in a manpower system, where individuals move through a network of states which may be defined in terms of ranks or position. One of the widely used approaches to the modeling of manpower systems is the Markov chain framework [1, 7, 9]. The basic Markov chain model for a $k$-grade manpower system is expressed algebraically using the following recursive relation

$$
n_{j}(t+1)=\sum_{i=1}^{k} n_{i}(t) p_{i j}+R(t+1) r_{j}, \quad j=1,2, \cdots, k,
$$

COPYRIGHT (c) by Publishing House of Rzeszów University of Technology P.O. Box 85, 35-959 Rzeszów, Poland 
where $n_{i}(t)$ is the expected number of individuals in state $i$ at time $t, p_{i j}$ is the internal homogeneous transition probability from state $i$ to state $j, r_{j}$ is the proportion of recruits allocated to state $j$ and $R(t+1)$ is the expected number of recruits to the system at time $t+1$. The manpower accounts for the system are assumed to take place at the end of the time period and recruitment is recorded as if it took place at the beginning of the next time period [1]. The transition probabilities, $p_{i j}$ 's, are estimated based on data from observable variables using the maximum likelihood method [14]. In many practical instances, the transition probability, $p_{i j}$, satisfies the conditions: $\sum_{j=1}^{k} p_{i j} \leq 1, i \in S, p_{i j} \geq 0, i, j \in S$, where $S=\{1,2, \cdots, k\}$ is the set of mutually exclusive and collectively exhaustive states of the $k$-grade manpower system. The shortfall in the sum $\sum_{j=1}^{k} p_{i j} \leq 1$ is attributed to outgoing flows (wastage) from the system. With $w_{i}$ as the wastage from the system,

$$
\sum_{j=1}^{k} p_{i j}+w_{i}=1, \quad i \in S
$$

The recursive relation in equation (1.1) can be rewritten in matrix notation as

$$
\mathbf{n}(t+1)=\mathbf{n}(t) \mathbf{P}+R(t+1) \mathbf{r},
$$

where $\mathbf{n}(t)=\left[n_{1}(t), n_{2}(2), \cdots, n_{k}(t)\right]$ is the structure of the system at any given time $t, \mathbf{P}=\left(p_{i j}\right)$ is the homogeneous transition matrix and $\mathbf{r}=\left[r_{1}, r_{2}, \cdots, r_{k}\right]$ is the recruitment vector with $\sum_{j=1}^{k} r_{i}=1$. Let $\mathbf{w}=\left[w_{1}, w_{2}, \cdots, w_{k}\right]$ denote the wastage vector for the system. Since a fixed size manpower system is considered, where wastage is replaced by new recruits, the expected number of recruits to the system at time $t+1$ is

$$
R(t+1)=\mathbf{n}(t) \mathbf{w}^{\prime} .
$$

Thus, equation (1.3) can be expressed as

$$
\mathbf{n}(t+1)=\mathbf{n}(t)\left(\mathbf{P}+\mathbf{w}^{\prime} \mathbf{r}\right)
$$

where $\left(\mathbf{P}+\mathbf{w}^{\prime} \mathbf{r}\right)$ is a stochastic matrix. Equation (1.5) is suitable to predict what the manpower structure will become one-step ahead year after year. If the manpower structure is to be maintained, then $\mathbf{n}(t+1)=\mathbf{n}(t)=\mathbf{n}$ in equation (1.5), cf. [13].

Suppose for motivational reasons, that the manpower structure is to be projected for a semester beyond one-step (that is, one year and six months) or a quarter beyond one-step (that is, one year and three months). Then representation becomes an issue when we have the fractional indicial stochastic matrix, $\left(\mathbf{P}+\mathbf{w}^{\prime} \mathbf{r}\right)^{1+1 / n}$, for $n=2$ or 4. This problem is an embeddability problem. Singer and Spilerman [11] considered the embeddability problem by verifying whether an observed transition matrix could have arisen from the evolution of a stationary continuous-time Markov process. The approach does not give a unique solution. Osagiede and Ekhosuehi [10] solved the embeddability problem for a manpower system with sparse stochastic matrices within the context of determining the nearest Markov generator arising from the continuoustime Markov chain to the higher order observable Markov chain. The resulting Markov 
chain was an approximation to the higher order observable Markov chain. In [6], the problem was solved by finding the diagonalizable form of the observable Markov chain.

This study considers a three-grade manpower system, that is, $k=3$. Markovian manpower systems with three grades arise in many practical situations $[1,3,4,7,8$, 13]. Following [12], the study assumes a fixed size manpower system that operates a policy that allows wastage to be replaced by new recruits. In this case, the consequential outflow from state $i$ which goes back to state $j$ as recruitment would be $w_{i} r_{j}, i, j \in S$. The study is aimed at finding the fractional indicial stochastic matrix, $\left(\mathbf{P}+\mathbf{w}^{\prime} \mathbf{r}\right)^{1+1 / n}$, arising from a hierarchical manpower system with three grades using the generating function technique (the so called z-transform). This approach that is based on z-transform has been used to model population dynamics within the Leslie matrices framework [2]. The study develops an additive representation for the stochastic matrix describing the evolution of the personnel structure of a Markov manpower system with fixed total size. The assumption of a fixed total size for manpower system is appropriate in practice when an organization is faced with limited personnel availability on the external labour market, facility and budget restrictions [8]. The usefulness of the additive representation is justified when there is a lack of observations regarding the time unit of the Markov chain (that was earlier estimated using historical data in discrete time) owing to a policy change in the short-term on the effective date of promotion. For instance, extending the effective date of promotion from October 1 of the current year to January 1 of the following year for budgetary reasons. This kind of policy change is dealt with in the additive representation.

\section{The generating function standpoint}

In this section, we prove the following using the z-transform: If $\mathbf{Q}=\left(\mathbf{P}+\mathbf{w}^{\prime} \mathbf{r}\right) \in \mathbb{R}^{3 \times 3}$ is a stochastic matrix that satisfies the axioms that: (i) $\mathbf{Q}$ is irreducible, (ii) the determinant of $\mathbf{Q}$ is non-singular, and (iii) the characteristic polynomial arising from the determinant $\operatorname{det}(\mathbf{I}-\mathbf{Q} z)$ has linear factors, then the fractional indicial stochastic matrix, $\boldsymbol{\Gamma}=\mathbf{Q}^{1+1 / n}, n>0$, can be expressed in the form

$$
\begin{gathered}
\boldsymbol{\Gamma}=\left\{\mathbf{X}=\left(x_{i j}\right) \in \mathbb{R}^{3 \times 3} \mid \mathbf{X}=\mathbf{A}_{m}+\mathbf{T}_{m}(1+1 / n),\right. \\
\left.\sum_{j=1}^{3} x_{i j}=1, x_{i j} \geq 0, \forall i, j \in S, m=1,2\right\},
\end{gathered}
$$

where $\mathbf{A}_{m}$ is the $3 \times 3$ matrix of limiting-state probabilities for case $m$ and

$$
\mathbf{T}_{m}(1+1 / n)=\left\{\begin{array}{cc}
\alpha_{1}^{-(2+1 / n)} \mathbf{B}_{1}+\alpha_{2}^{-(2+1 / n)} \mathbf{C}, \quad m=1 \text { if }(\operatorname{tr}(\mathbf{Q})-1)^{2}>4 \operatorname{det}(\mathbf{Q}) \\
(2+1 / n) \alpha^{-(3+1 / n)} \mathbf{B}_{2}+\alpha^{-(2+1 / n)} \mathbf{D}, \quad m=2 \text { if }(\operatorname{tr}(\mathbf{Q})-1)^{2}=4 \operatorname{det}(\mathbf{Q})
\end{array}\right.
$$

provided that $\alpha, \alpha_{1}, \alpha_{2} \in \Psi=\{v \mid v>1, v \in \mathbb{R}\}$ with $\alpha, \alpha_{1}, \alpha_{2}$ being the zeros of the characteristic function $\operatorname{det}(\mathbf{I}-\mathbf{Q} z)=1-\operatorname{tr}(\mathbf{Q}) z+\left(\sum_{i=1}^{3} Q_{i i}\right) z^{2}-\operatorname{det}(\mathbf{Q}) z^{3}$ with 
$Q_{i i}$ being the cofactor of the diagonal entries in $\mathbf{Q}$, and $\mathbf{B}_{m}, \mathbf{C}, \mathbf{D}$ are matrices of constant values for each respective case $m$.

Consider the recurrence relation in equation (1.5): Using the z-transform, the generation function vector $\mathbf{g}(z)$ that is associated with the manpower structure $\mathbf{n}(t)$ is defined by

$$
\mathbf{g}(z)=\sum_{t=0}^{\infty} \mathbf{n}(t) z^{t}
$$

Thus,

$$
\mathbf{g}(z) \mathbf{Q}=\sum_{t=0}^{\infty} \mathbf{n}(t) \mathbf{Q} z^{t}=\sum_{t=0}^{\infty} \mathbf{n}(t+1) z^{t}=\frac{1}{z} \sum_{t=0}^{\infty} \mathbf{n}(t+1) z^{t+1}=\frac{1}{z}(\mathbf{g}(z)-\mathbf{n}(0)),
$$

where $\mathbf{n}(0)$ is the initial manpower structure. Further simplifications lead to

$$
\mathbf{g}(z)=\mathbf{n}(0)[\mathbf{I}-\mathbf{Q} z]^{-1} .
$$

Let

$$
\mathbf{G}(z)=[\mathbf{I}-\mathbf{Q} z]^{-1}=\sum_{t=0}^{\infty} \mathbf{Q}^{t} z^{t}, \quad \mathbf{Q}^{0}=\mathbf{I},
$$

where $\mathbf{G}(z)$ is the $3 \times 3$ Green function matrix and $\mathbf{I}$ is the $3 \times 3$ identity matrix. Since

$$
\mathbf{Q}=\left[\begin{array}{lll}
p_{11} & p_{12} & p_{13} \\
p_{21} & p_{22} & p_{23} \\
p_{31} & p_{32} & p_{33}
\end{array}\right]+\left[\begin{array}{l}
w_{1} \\
w_{2} \\
w_{3}
\end{array}\right]\left[\begin{array}{lll}
r_{1} & r_{2} & r_{3}
\end{array}\right]=\left(q_{i j}\right)
$$

where $q_{i j}=p_{i j}+w_{i} r_{j}, i, j \in S$, then

$$
\mathbf{I}-\mathbf{Q} z=\left[\begin{array}{ccc}
1-q_{11} z & -q_{12} z & -q_{13} z \\
-q_{21} z & 1-q_{22} z & -q_{23} z \\
-q_{31} z & -q_{32} z & 1-q_{33} z
\end{array}\right]
$$

The inverse of $\mathbf{I}-\mathbf{Q} z$ is defined as

$$
[\mathbf{I}-\mathbf{Q} z]^{-1}=\frac{\operatorname{adj}(\mathbf{I}-\mathbf{Q} z)}{\operatorname{det}(\mathbf{I}-\mathbf{Q} z)} .
$$

The determinant, $\operatorname{det}(\mathbf{I}-\mathbf{Q} z)$, is obtained as follows: Factorizing $\left(1-q_{11} z\right), q_{12} z$, $q_{13} z$ from column 1, 2, 3 respectively of $\operatorname{det}(\mathbf{I}-\mathbf{Q} z)$ yields

$$
\operatorname{det}(\mathbf{I}-\mathbf{Q} z)=\left(1-q_{11} z\right) q_{12} q_{13} z^{2}\left|\begin{array}{ccc}
1 & -1 & -1 \\
-\frac{q_{21} z}{\left(1-q_{11} z\right)} & \frac{1-q_{22} z}{q_{12} z} & -\frac{q_{23}}{q_{13}} \\
-\frac{q_{31} z}{\left(1-q_{11} z\right)} & -\frac{q_{32}}{q_{12}} & \frac{1-q_{33} z}{q_{13} z}
\end{array}\right| .
$$

Subtracting column 2 from column 3, we have

$$
\operatorname{det}(\mathbf{I}-\mathbf{Q} z)=\left(1-q_{11} z\right) q_{12} q_{13} z^{2}\left|\begin{array}{ccc}
1 & -1 & 0 \\
-\frac{q_{21} z}{\left(1-q_{11} z\right)} & \frac{1-q_{22} z}{q_{12} z} & -\frac{q_{23}}{q_{13}}-\frac{1-q_{22} z}{q_{12} z} \\
-\frac{q_{31} z}{\left(1-q_{11} z\right)} & -\frac{q_{32}}{q_{12}} & \frac{1-q_{33} z}{q_{13} z}+\frac{q_{32}}{q_{12}}
\end{array}\right| .
$$


Adding column 1 to column 2,

$$
\operatorname{det}(\mathbf{I}-\mathbf{Q} z)=\left(1-q_{11} z\right) q_{12} q_{13} z^{2}\left|\begin{array}{ccc}
1 & 0 & 0 \\
-\frac{q_{21} z}{\left(1-q_{11} z\right)} & \frac{1-q_{22} z}{q_{12} z}-\frac{q_{21} z}{\left(1-q_{11} z\right)} & -\frac{q_{23}}{q_{13}}-\frac{1-q_{22} z}{q_{12} z} \\
-\frac{q_{31} z}{\left(1-q_{11} z\right)} & -\frac{q_{32}}{q_{12}}-\frac{q_{31} z}{\left(1-q_{11} z\right)} & \frac{1-q_{33} z}{q_{13} z}+\frac{q_{32}}{q_{12}}
\end{array}\right| .
$$

Taking the determinant

$$
\begin{array}{r}
\operatorname{det}(\mathbf{I}-\mathbf{Q} z)=\left(1-q_{11} z\right) q_{12} q_{13} z^{2}\left(\left(\frac{1-q_{22} z}{q_{12} z}-\frac{q_{21} z}{\left(1-q_{11} z\right)}\right)\left(\frac{1-q_{33} z}{q_{13} z}+\frac{q_{32}}{q_{12}}\right)-\right. \\
\left.\left(\frac{q_{23}}{q_{13}}-\frac{1-q_{22} z}{q_{12} z}\right)\left(\frac{q_{32}}{q_{12}}-\frac{q_{31} z}{\left(1-q_{11} z\right)}\right)\right) .
\end{array}
$$

This simplifies to

$$
\begin{array}{r}
\operatorname{det}(\mathbf{I}-\mathbf{Q} z)=1-\left(q_{11}+q_{22}+q_{33}\right) z+\left(q_{11} q_{22}+q_{11} q_{33}+q_{22} q_{33}-q_{21} q_{12}-q_{23} q_{32}-q_{31} q_{13}\right) z^{2} \\
-\left(q_{11} q_{22} q_{33}-q_{21} q_{12} q_{33}+q_{21} q_{32} q_{13}-q_{23} q_{11} q_{32}+q_{23} q_{12} q_{31}-q_{13} q_{22} q_{31}\right) z^{3}
\end{array}
$$

Thus

$$
\operatorname{det}(\mathbf{I}-\mathbf{Q} z)=1-\operatorname{tr}(\mathbf{Q}) z+\left(\sum_{i=1}^{3} Q_{i i}\right) z^{2}-\operatorname{det}(\mathbf{Q}) z^{3}
$$

Now $(1-z)$ is a factor of the cubic characteristic function (2.5) since at $z=1$,

$$
1-\operatorname{tr}(\mathbf{Q})+\left(\sum_{i=1}^{3} Q_{i i}\right)-\operatorname{det}(\mathbf{Q})=\left|\begin{array}{ccc}
1-q_{11} & -q_{12} & -q_{13} \\
-q_{21} & 1-q_{22} & -q_{23} \\
-q_{31} & -q_{32} & 1-q_{33}
\end{array}\right|
$$

Equation (2.6) simplifies to

$$
\left|\begin{array}{ccc}
q_{12}+q_{13} & -q_{12} & -q_{13} \\
-q_{21} & q_{21}+q_{23} & -q_{23} \\
-q_{31} & -q_{32} & q_{31}+q_{32}
\end{array}\right|=\left|\begin{array}{ccc}
q_{13} & -q_{12} & -q_{13} \\
q_{23} & q_{21}+q_{23} & -q_{23} \\
-\left(q_{31}+q_{32}\right) & -q_{32} & q_{31}+q_{32}
\end{array}\right|=0
$$

as column 1 and column 3 are identical. It follows that

$$
\operatorname{det}(\mathbf{I}-\mathbf{Q} z)=(1-z)\left(1-(\operatorname{tr}(\mathbf{Q})-1) z+\operatorname{det}(\mathbf{Q}) z^{2}\right) .
$$

Using the fundamental theorem of algebra, equation (2.7) is expressed as

$$
\operatorname{det}(\mathbf{I}-\mathbf{Q} z)=\operatorname{det}(\mathbf{Q})(1-z)\left(\alpha_{1}-z\right)\left(\alpha_{2}-z\right)
$$

where

$$
\alpha_{1}=\frac{\operatorname{tr}(\mathbf{Q}-1)}{2 \operatorname{det}(\mathbf{Q})}\left(1-\left(1-\frac{4 \operatorname{det}(\mathbf{Q})}{(\operatorname{tr}(\mathbf{Q})-1)^{2}}\right)^{1 / 2}\right)
$$


and

$$
\alpha_{2}=\frac{\operatorname{tr}(\mathbf{Q}-1)}{2 \operatorname{det}(\mathbf{Q})}\left(1+\left(1-\frac{4 \operatorname{det}(\mathbf{Q})}{(\operatorname{tr}(\mathbf{Q})-1)^{2}}\right)^{1 / 2}\right),
$$

provided that $\operatorname{det}(\mathbf{Q}) \neq 0$. The roots $\alpha_{1}$ and $\alpha_{2}$ are real if $(\operatorname{tr}(\mathbf{Q})-1)^{2} \geq 4 \operatorname{det}(\mathbf{Q})$. If $(\operatorname{tr}(\mathbf{Q})-1)^{2}<4 \operatorname{det}(\mathbf{Q}), \alpha_{1}$ and $\alpha_{2}$ would produce complex entries and these have no meaning within the context of Markov chains. Thus, the case where the quadratic form $\left(1-(\operatorname{tr}(\mathbf{Q})-1) z+\operatorname{det}(\mathbf{Q}) z^{2}\right)$ does not have linear factors is not considered. Moreover, it is difficult to simplify the reciprocal of $\left(1-(\operatorname{tr}(\mathbf{Q})-1) z+\operatorname{det}(\mathbf{Q}) z^{2}\right)$ as a series in the form $\sum_{r=0}^{\infty} \theta^{r} z^{r}$, where $\theta$ is independent of $z$. More specifically,

$\frac{1}{\left(1-(\operatorname{tr}(\mathbf{Q})-1) z+\operatorname{det}(\mathbf{Q}) z^{2}\right)}=\sum_{r=0}^{\infty}\left(\sum_{s=0}^{r}(-1)^{s}\left(\begin{array}{l}r \\ s\end{array}\right)(\operatorname{det}(\mathbf{Q}))^{s}(\operatorname{tr}(\mathbf{Q})-1)^{r-s} z^{s}\right) z^{r}$

However, the reciprocal of each of the factors in equation (2.8) when $\alpha_{1}$ and $\alpha_{2}$ are real can be expressed in the following series

$$
\begin{gathered}
\frac{1}{1-z}=\sum_{t=0}^{\infty} z^{t} . \\
\frac{1}{\alpha-z}=\sum_{t=0}^{\infty} \alpha^{-(1+t)} z^{t} . \\
\frac{1}{(\alpha-z)^{2}}=\sum_{t=0}^{\infty}(1+t) \alpha^{-(2+t)} z^{t} .
\end{gathered}
$$

To obtain the $\operatorname{adj}(\mathbf{I}-\mathbf{Q} z)$, we first find the cofactors of each entry in $(\mathbf{I}-\mathbf{Q} z)$. The cofactor of $1-q_{11} z$ is $\Lambda_{11}(z)=1-\left(q_{22}+q_{33}\right) z+\left(q_{22} q_{33}-q_{23} q_{32}\right) z^{2}$, the cofactor of $-q_{12} z$ is $\Lambda_{12}(z)=q_{21} z-\left(q_{21} q_{33}-q_{23} q_{31}\right) z^{2}$ and so on. Proceeding in this way, the entries in the $\operatorname{adj}(\mathbf{I}-\mathbf{Q} z)$ are found to be a polynomial in $z$ of degree two. More precisely,

$$
\operatorname{adj}(\mathbf{I}-\mathbf{Q} z)=\left[\begin{array}{ccc}
\Lambda_{11}(z) & \Lambda_{21}(z) & \Lambda_{31}(z) \\
\Lambda_{12}(z) & \Lambda_{22}(z) & \Lambda_{32}(z) \\
\Lambda_{13}(z) & \Lambda_{23}(z) & \Lambda_{33}(z)
\end{array}\right],
$$

where $\Lambda_{13}(z)=q_{31} z+\left(q_{21} q_{32}-q_{22} q_{31}\right) z^{2}, \Lambda_{21}(z)=q_{12} z+\left(q_{13} q_{32}-q_{12} q_{33}\right) z^{2}, \Lambda_{22}(z)=$ $1-\left(q_{11}+q_{33}\right) z+\left(q_{11} q_{33}-q_{13} q_{31}\right) z^{2}, \Lambda_{23}(z)=q_{32} z-\left(q_{11} q_{32}-q_{12} q_{31}\right) z^{2}, \Lambda_{31}(z)=$ $q_{13} z+\left(q_{12} q_{23}-q_{13} q_{22}\right) z^{2}, \Lambda_{32}(z)=q_{23} z-\left(q_{11} q_{23}-q_{13} q_{21}\right) z^{2}$ and $\Lambda_{33}(z)=1-\left(q_{11}+\right.$ $\left.q_{22}\right) z+\left(q_{11} q_{22}-q_{12} q_{21}\right) z^{2}$.

Resolving the quotient (2.4) into the sum of partial fractions and using the expressions (2.9) to (2.11), we obtain the following results for each case $m$ according to whether $(\operatorname{tr}(\mathbf{Q})-1)^{2}>4 \operatorname{det}(\mathbf{Q})$ or $(\operatorname{tr}(\mathbf{Q})-1)^{2}=4 \operatorname{det}(\mathbf{Q})$. 


\section{Case 1}

If $(\operatorname{tr}(\mathbf{Q})-1)^{2}>4 \operatorname{det}(\mathbf{Q})$, then

$$
\begin{aligned}
{[\mathbf{I}-\mathbf{Q} z]^{-1}=} & \sum_{t=0}^{\infty} \frac{1}{\operatorname{det}(\mathbf{Q})}\left(\frac{1}{\left(\alpha_{1}-1\right)\left(\alpha_{2}-1\right)}\left[\begin{array}{lll}
a_{11} & a_{12} & a_{13} \\
a_{21} & a_{22} & a_{23} \\
a_{31} & a_{32} & a_{33}
\end{array}\right]+\frac{\alpha_{1}^{-(1+t)}}{\left(\alpha_{1}-1\right)\left(\alpha_{1}-\alpha_{2}\right)} \times\right. \\
& {\left.\left[\begin{array}{lll}
b_{11} & b_{12} & b_{13} \\
b_{21} & b_{22} & b_{23} \\
b_{31} & b_{32} & b_{33}
\end{array}\right]+\frac{\alpha_{2}^{-(1+t)}}{\left(\alpha_{2}-1\right)\left(\alpha_{2}-\alpha_{1}\right)}\left[\begin{array}{lll}
c_{11} & c_{12} & c_{13} \\
c_{21} & c_{22} & c_{23} \\
c_{31} & c_{32} & c_{33}
\end{array}\right]\right) z^{t}, \quad(2.12) }
\end{aligned}
$$

where $a_{11}=1-\left(q_{22}+q_{33}\right)+\left(q_{22} q_{33}-q_{23} q_{32}\right), a_{12}=q_{12}+\left(q_{13} q_{32}-q_{12} q_{33}\right), a_{13}=q_{13}+$ $\left(q_{12} q_{23}-q_{13} q_{22}\right), a_{21}=q_{21}-\left(q_{21} q_{33}-q_{23} q_{31}\right), a_{22}=1-\left(q_{11}+q_{33}\right)+\left(q_{11} q_{33}-q_{13} q_{31}\right)$, $a_{23}=q_{23}-\left(q_{11} q_{23}-q_{13} q_{21}\right), a_{31}=q_{31}+\left(q_{21} q_{32}-q_{22} q_{31}\right), a_{32}=q_{32}-\left(q_{11} q_{32}-q_{12} q_{31}\right)$, $a_{33}=1-\left(q_{11}+q_{22}\right)+\left(q_{11} q_{22}-q_{12} q_{21}\right), b_{11}=1-\left(q_{22}+q_{33}\right) \alpha_{1}+\left(q_{22} q_{33}-q_{23} q_{32}\right) \alpha_{1}^{2}$, $b_{21}=q_{12} \alpha_{1}+\left(q_{13} q_{32}-q_{12} q_{33}\right) \alpha_{1}^{2}, b_{31}=q_{13} \alpha_{1}+\left(q_{12} q_{23}-q_{13} q_{22}\right) \alpha_{1}^{2}, b_{12}=q_{21} \alpha_{1}-$ $\left(q_{21} q_{33}-q_{23} q_{31}\right) \alpha_{1}^{2}, b_{22}=1-\left(q_{11}+q_{33}\right) \alpha_{1}+\left(q_{11} q_{33}-q_{13} q_{31}\right) \alpha_{1}^{2}, b_{32}=q_{23} \alpha_{1}-$ $\left(q_{11} q_{23}-q_{13} q_{21}\right) \alpha_{1}^{2}, b_{13}=q_{31} \alpha_{1}+\left(q_{21} q_{32}-q_{22} q_{31}\right) \alpha_{1}^{2}, b_{23}=q_{32} \alpha_{1}-\left(q_{11} q_{32}-q_{12} q_{31}\right) \alpha_{1}^{2}$, $b_{33}=1-\left(q_{11}+q_{22}\right) \alpha_{1}+\left(q_{11} q_{22}-q_{12} q_{21}\right) \alpha_{1}^{2}, c_{11}=1-\left(q_{22}+q_{33}\right) \alpha_{2}+\left(q_{22} q_{33}-q_{23} q_{32}\right) \alpha_{2}^{2}$, $c_{21}=q_{12} \alpha_{2}+\left(q_{13} q_{32}-q_{12} q_{33}\right) \alpha_{2}^{2}, c_{31}=q_{13} \alpha_{2}+\left(q_{12} q_{23}-q_{13} q_{22}\right) \alpha_{2}^{2}, c_{12}=q_{21} \alpha_{2}-$ $\left(q_{21} q_{33}-q_{23} q_{31}\right) \alpha_{2}^{2}, c_{22}=1-\left(q_{11}+q_{33}\right) \alpha_{2}+\left(q_{11} q_{33}-q_{13} q_{31}\right) \alpha_{2}^{2}, c_{32}=q_{23} \alpha_{2}-$ $\left(q_{11} q_{23}-q_{13} q_{21}\right) \alpha_{2}^{2}, c_{13}=q_{31} \alpha_{2}+\left(q_{21} q_{32}-q_{22} q_{31}\right) \alpha_{2}^{2}, c_{23}=q_{32} \alpha_{2}-\left(q_{11} q_{32}-q_{12} q_{31}\right) \alpha_{2}^{2}$, $c_{33}=1-\left(q_{11}+q_{22}\right) \alpha_{2}+\left(q_{11} q_{22}-q_{12} q_{21}\right) \alpha_{2}^{2}$.

\section{Case 2}

If $(\operatorname{tr}(\mathbf{Q})-1)^{2}=4 \operatorname{det}(\mathbf{Q})$, then $\alpha_{1}=\alpha_{2}=\alpha$ and

$$
\begin{aligned}
{[\mathbf{I}-\mathbf{Q} z]^{-1}=} & \sum_{t=0}^{\infty}\left(\frac{1}{(\alpha-1)^{2} \operatorname{det}(\mathbf{Q})}\left[\begin{array}{lll}
a_{11} & a_{12} & a_{13} \\
a_{21} & a_{22} & a_{23} \\
a_{31} & a_{32} & a_{33}
\end{array}\right]+\frac{(1+t) \alpha^{-(2+t)}}{(\alpha-1) \operatorname{det}(\mathbf{Q})} \times\right. \\
& {\left.\left[\begin{array}{lll}
b_{11} & b_{12} & b_{13} \\
b_{21} & b_{22} & b_{23} \\
b_{31} & b_{32} & b_{33}
\end{array}\right]+\frac{\alpha^{-(1+t)}}{\alpha}\left[\begin{array}{lll}
d_{11} & d_{12} & d_{13} \\
d_{21} & d_{22} & d_{23} \\
d_{31} & d_{32} & d_{33}
\end{array}\right]\right) z^{t} }
\end{aligned}
$$

where $d_{11}=\left(1 / \operatorname{det}(\mathbf{Q})-\alpha^{2} a_{11}-b_{11}\right), d_{12}=-\left(\alpha^{2} a_{12}+b_{12}\right), d_{13}=-\left(\alpha^{2} a_{13}+b_{13}\right)$, $d_{21}=-\left(\alpha^{2} a_{21}+b_{21}\right), d_{22}=\left(1 / \operatorname{det}(\mathbf{Q})-\alpha^{2} a_{22}-b_{22}\right), d_{23}=-\left(\alpha^{2} a_{23}+b_{23}\right)$, $d_{31}=-\left(\alpha^{2} a_{31}+b_{31}\right), d_{32}=-\left(\alpha^{2} a_{32}+b_{32}\right), d_{33}=\left(1 / \operatorname{det}(\mathbf{Q})-\alpha^{2} a_{33}-b_{33}\right)$.

In the expression for Case 1 , let

$$
\mathbf{A}_{1}=\frac{1}{\left(\alpha_{1}-1\right)\left(\alpha_{2}-1\right) \operatorname{det}(\mathbf{Q})}\left[\begin{array}{lll}
a_{11} & a_{12} & a_{13} \\
a_{21} & a_{22} & a_{23} \\
a_{31} & a_{32} & a_{33}
\end{array}\right],
$$




$$
\mathbf{B}_{1}=\frac{1}{\left(\alpha_{1}-1\right)\left(\alpha_{1}-\alpha_{2}\right) \operatorname{det}(\mathbf{Q})}\left[\begin{array}{lll}
b_{11} & b_{12} & b_{13} \\
b_{21} & b_{22} & b_{23} \\
b_{31} & b_{32} & b_{33}
\end{array}\right]
$$

and

$$
\mathbf{C}=\frac{1}{\left(\alpha_{2}-1\right)\left(\alpha_{2}-\alpha_{1}\right) \operatorname{det}(\mathbf{Q})}\left[\begin{array}{ccc}
c_{11} & c_{12} & c_{13} \\
c_{21} & c_{22} & c_{23} \\
c_{31} & c_{32} & c_{33}
\end{array}\right]
$$

and for Case 2, let

$$
\begin{gathered}
\mathbf{A}_{2}=\frac{1}{(\alpha-1)^{2} \operatorname{det}(\mathbf{Q})}\left[\begin{array}{lll}
a_{11} & a_{12} & a_{13} \\
a_{21} & a_{22} & a_{23} \\
a_{31} & a_{32} & a_{33}
\end{array}\right], \\
\mathbf{B}_{2}=\frac{1}{(\alpha-1) \operatorname{det}(\mathbf{Q})}\left[\begin{array}{lll}
b_{11} & b_{12} & b_{13} \\
b_{21} & b_{22} & b_{23} \\
b_{31} & b_{32} & b_{33}
\end{array}\right]
\end{gathered}
$$

and

$$
\mathbf{D}=\frac{1}{\alpha}\left[\begin{array}{lll}
d_{11} & d_{12} & d_{13} \\
d_{21} & d_{22} & d_{23} \\
d_{31} & d_{32} & d_{33}
\end{array}\right]
$$

Making the appropriate substitution for $[\mathbf{I}-\mathbf{Q} z]^{-1}$, it follows from equation (2.1) for any given $t=1+1 / n, n>0$, that

$$
\mathbf{Q}^{(1+1 / n)}=\mathbf{A}_{m}+\mathbf{T}_{m}(1+1 / n), \quad m=1,2,
$$

where

$\mathbf{T}_{m}(1+1 / n)=\left\{\begin{array}{cc}\alpha_{1}^{-(2+1 / n)} \mathbf{B}_{1}+\alpha_{2}^{-(2+1 / n)} \mathbf{C}, \quad m=1 \text { if }(\operatorname{tr}(\mathbf{Q})-1)^{2}>4 \operatorname{det}(\mathbf{Q}) \\ (2+1 / n) \alpha^{-(3+1 / n)} \mathbf{B}_{2}+\alpha^{-(2+1 / n)} \mathbf{D}, & m=2 \text { if }(\operatorname{tr}(\mathbf{Q})-1)^{2}=4 \operatorname{det}(\mathbf{Q}) .\end{array}\right.$

As $\mathbf{Q}$ is irreducible, it follows for large $t$ that

$$
\lim _{t \rightarrow \infty} \mathbf{Q}^{t}=\mathbf{A}_{m}+\lim _{t \rightarrow \infty} \mathbf{T}_{m}(t)
$$

exists. This would hold only if $\alpha_{1}, \alpha_{2}>1$. With $\alpha_{1}, \alpha_{2}>1, \lim _{t \rightarrow \infty} \mathbf{T}_{m}(t)=\mathbf{0}$. In either case $m, \mathbf{A}_{m}$ is a matrix of limiting-state probabilities.

To show that the matrix $\mathbf{Q}^{t}$ is meaningful for any given $t=1+1 / n, n>0$, if $\alpha_{1}, \alpha_{2}>1$, consider the doubly stochastic matrix in [5]:

$$
\mathbf{P}+\mathbf{w}^{\prime} \mathbf{r}=\left[\begin{array}{ccc}
0.5 & 0.5 & 0 \\
0.5 & 0.25 & 0.25 \\
0 & 0.25 & 0.75
\end{array}\right]
$$


which has the real roots $\alpha_{1}=1.4641$ and $\alpha_{2}=-5.4641$. The additive representation is

$$
\begin{array}{r}
\mathbf{Q}^{1+1 / n}=\left[\begin{array}{lll}
0.3333 & 0.3333 & 0.3333 \\
0.3333 & 0.3333 & 0.3333 \\
0.3333 & 0.3333 & 0.3333
\end{array}\right]+(1.4641)^{-(2+1 / n)}\left[\begin{array}{ccc}
0.4880 & 0.1786 & -0.6667 \\
0.1786 & 0.0654 & -0.2440 \\
-0.6667 & -0.2440 & 0.9107
\end{array}\right] \\
+(-5.4641)^{-(2+1 / n)}\left[\begin{array}{ccc}
-1.8214 & 2.4880 & -0.6667 \\
2.4880 & -3.3987 & 0.9107 \\
-0.6667 & 0.9107 & -0.2440
\end{array}\right] .
\end{array}
$$

For any $n>0$, the third term is a matrix of complex entries because the $n$th root, $(-5.4641)^{1 / n}$, arising from the scalar $(-5.4641)^{-(2+1 / n)}$, does not exist. Thus the fractional indicial matrix $\left(\mathbf{P}+\mathbf{w}^{\prime} \mathbf{r}\right)^{(1+1 / n)}$ cannot be represented as a sum of constant matrices that is meaningful within the Markov chain framework.

\section{Illustration}

The applicability of the new representation for the irreducible stochastic matrix $\mathbf{Q}$ is demonstrated in this section. We consider two test problems. The first problem is contained in [11] and the second one is in [12].

Example 1. Singer and Spilerman [11] expressed the following transition matrix

$$
\tilde{\mathbf{P}}=\left[\begin{array}{ccc}
0.16 & 0.53 & 0.31 \\
0.0525 & 0.49 & 0.4575 \\
0.11 & 0.14 & 0.75
\end{array}\right]
$$

in terms of the intensity matrix as

$$
\hat{\mathbf{P}}=\exp \left(\left[\begin{array}{ccc}
-2.046 & 1.993 & 0.053 \\
0.024 & -0.818 & 0.794 \\
0.315 & 0.043 & -0.358
\end{array}\right]\right)
$$

where $\hat{\mathbf{P}}$ is an embeddable matrix of $\tilde{\mathbf{P}}$. Clearly, $\hat{\mathbf{P}}$ is an approximation of $\tilde{\mathbf{P}}$ as

$$
\hat{\mathbf{P}}=\exp \left(\left[\begin{array}{ccc}
-2.046 & 1.993 & 0.053 \\
0.024 & -0.818 & 0.794 \\
0.315 & 0.043 & -0.358
\end{array}\right]\right)=\left[\begin{array}{ccc}
0.1601 & 0.5296 & 0.3103 \\
0.0525 & 0.4894 & 0.4581 \\
0.1105 & 0.1405 & 0.7489
\end{array}\right]
$$

The additive representation is possible as $\operatorname{det}(\tilde{\mathbf{P}})=0.0399$ is non-singular, the difference $(\operatorname{tr}(\tilde{\mathbf{P}})-1)^{2}-4 \operatorname{det}(\tilde{\mathbf{P}})=0.16-0.1597>0$, and the roots of the determinant $\operatorname{det}(\mathbf{I}-\tilde{\mathbf{P}} z)$ are real and greater than one, viz.

$$
\alpha_{1}=\frac{0.4}{2(0.0399)}\left(1-\left(1-\frac{4(0.0399)}{(0.4)^{2}}\right)^{1 / 2}\right)=4.7925
$$


and

$$
\alpha_{2}=\frac{0.4}{2(0.0399)}\left(1+\left(1-\frac{4(0.0399)}{(0.4)^{2}}\right)^{1 / 2}\right)=5.2263 .
$$

Using the additive representation, the $(1+1 / n)$-step transition matrix, $\mathbf{Q}^{(1+1 / n)}$, for $n>0$, is represented as:

$$
\begin{aligned}
\mathbf{Q}^{1+1 / n} & =\left[\begin{array}{lll}
0.0992 & 0.2749 & 0.6260 \\
0.0992 & 0.2749 & 0.6260 \\
0.0992 & 0.2749 & 0.6260
\end{array}\right] \\
& +(4.7925)^{-(2+1 / n)}\left[\begin{array}{ccc}
-30.8570 & 85.1439 & -54.2869 \\
-7.6590 & 21.1336 & -13.4746 \\
8.2509 & -22.7667 & 14.5158
\end{array}\right] \\
+ & (5.2263)^{-(2+1 / n)}\left[\begin{array}{ccc}
38.3583 & -94.2879 & 55.9296 \\
7.8341 & -19.2569 & 11.4228 \\
-9.5160 & 23.3910 & -13.8751
\end{array}\right] .
\end{aligned}
$$

This representation does not require any form of perturbation as $\mathbf{Q}$ is equal to $\tilde{\mathbf{P}}$.

Example 2. Tsaklidis [12] considered a continuous time homogeneous Markov system with fixed size, where the matrix of the transition intensities of the memberships is given as

$$
\boldsymbol{\Phi}=\left[\begin{array}{ccc}
-1 / 2 & 0 & 1 / 2 \\
1 / 8 & -1 / 2 & 3 / 8 \\
0 & 1 / 2 & -1 / 2
\end{array}\right]
$$

In this example, the $\operatorname{determinant} \operatorname{det}(\mathbf{I}-z \exp (\mathbf{\Phi}))$ has equal roots, that is, $\alpha_{1}=$ $\alpha_{2}=2.1170$. We obtain a meaningful $(1+1 / n)$-step transition matrix for any given $n>0$, using the additive representation as:

$$
\begin{aligned}
\mathbf{Q}^{1+1 / n} & =\left[\begin{array}{lll}
0.1111 & 0.4444 & 0.4444 \\
0.1111 & 0.4444 & 0.4444 \\
0.1111 & 0.4444 & 0.4444
\end{array}\right] \\
& +(2+1 / n)(2.117)^{-(3+1 / n)}\left[\begin{array}{ccc}
0.7469 & -1.4939 & 0.7469 \\
0.1867 & -0.3735 & 0.1867 \\
-0.3735 & 0.7469 & -0.3735
\end{array}\right] \\
& +(2.117)^{-(2+1 / n)}\left[\begin{array}{ccc}
1.5289 & -0.2352 & -1.2937 \\
-0.3234 & 1.3525 & -1.0291 \\
-0.0588 & -1.2937 & 1.3525
\end{array}\right] .
\end{aligned}
$$

The matrix $\mathbf{Q}^{1+1 / n}$ is a stochastic matrix and is compatible with the continuous-time representation, $\exp ((1+1 / n) \boldsymbol{\Phi})$, for any given $n>0$.

Suppose that there exist an initial structure $\mathbf{n}(0)=[55,40,5]$. Then the results of using the additive representation for a shift in the unit interval of the Markov chain by 3 months, 6 months and 9 months are $\mathbf{n}(1+1 / 4)=[33,33,34], \mathbf{n}(1+1 / 2)=[30,33,37]$ 
and $\mathbf{n}(1+3 / 4)=[28,33,39]$, respectively ${ }^{1}$. These results are consistent with the continuous time process for $t=5 / 4,3 / 2,7 / 4$.

\section{Conclusion}

This paper has provided the additive representation of stochastic matrices as a means for obtaining fractional indicial matrices for the manpower system where the personnel structure is to be projected for a few months beyond one year (for instance, one year and six months, one year and three months, etc.). As an alternative to the assertion that supports the continuous-time formulation in place of the discrete-time Markov framework [11], this study gives instances where certain discrete-time Markov framework for forecasting manpower structure could have a meaningful fractional indicial stochastic matrix without recourse to the continuous-time representation via the transition intensities. The approach in this paper circumvents the problem of nonuniqueness that exists in the earlier formulations $[6,11]$. Even so three conditions should be satisfied: (i) the transition matrix $\mathbf{Q}$ is irreducible, (ii) the determinant of $\mathbf{Q}$ is non-singular, and (iii) the characteristic polynomial arising from the determinant $\operatorname{det}(\mathbf{I}-\mathbf{Q} z)$ has linear factors with real roots, which exceeds one. For instances where these conditions are violated, no substantive meaning can be attached in the additive context. In that case, the appropriate mathematical structure is a continuous-time formulation.

\section{References}

[1] D.J. Bartholomew, A.F. Forbes, S.I. McClean, Statistical Techniques for Manpower Planning, 2nd edn. John Wiley \& Sons, Chichester, 1991.

[2] M.O. Cacéres, I. Cacéres-Saez, Random Leslie matrices in population dynamics, Journal of Mathematical Biology 63 (2011) 519-556.

[3] V.U. Ekhosuehi, A control rule for planning promotion in a university setting in Nigeria, Croatian Operational Research Review 7 (2) (2016) 171-188.

[4] M.-A. Guerry, Monotonicity property of t-step maintainable structures in threegrade manpower systems: a counterexample, Journal of Applied Probability 28 (1) (1991) 221-224.

[5] M.-A. Guerry, Properties of calculated predictions of grade sizes and the associated integer valued vectors. Journal of Applied Probability 34 (1) (1997) 94-100.

[6] M.-A. Guerry, On the embedding problem for discrete-time Markov chains, Journal of Applied Probability 50 (4) (2013) 918-930.

\footnotetext{
${ }^{1}$ The results are approximated to the nearest integer without violating the assumption of a fixed total size.
} 
[7] M.-A. Guerry, T. De Feyter, Optimal recruitment strategies in a multi-level manpower planning model. Journal of the Operational Research Society 63 (2012), 931-940. DOI: 10.10.1057/jors.2011.99.

[8] Komarudin, M.-A. Guerry, G. Vanden Berghe, T. De Feyter, Balancing attainability, desirability and promotion steadiness in manpower planning systems, Journal of the Operational Research Society 66 (12) (2015) 2004-2014. DOI: 10.1057/jors.2015.26.

[9] K. Nilakantan, Evaluation of staffing policies in Markov manpower systems and their extension to organizations with outsource personnel, Journal of the Operational Research Society 66 (8) (2015) 1324-1340. DOI: 10.1057/jors.2014.82.

[10] A.A. Osagiede, V.U. Ekhosuehi, Finding a continuous-time Markov chain via sparse stochastic matrices in manpower systems, Journal of the Nigeria Mathematical Society 34 (2015) 94-105.

[11] B. Singer, S. Spilerman, The representation of social processes by Markov models, American Journal of Sociology 82 (1) (1976) 1-54.

[12] G.M. Tsaklidis, The evolution of the attainable structures of a continuous time homogeneous Markov system with fixed size, Journal of Applied Probability 33 (1) (1996) 34-47.

[13] A.U. Udom, Optimal controllability of manpower system with linear quadratic performance index, Brazilian Journal of Probability and Statistics 28 (2) (2014) 151-166.

[14] S.H. Zanakis, M.W. Maret, A Markov chain application to manpower supply planning, Journal of the Operational Research Society 31 (12) (1980) 1095-1102.

DOI: $10.7862 / \mathrm{rf} .2020 .1$

Vincent A. Amenaghawon

email: vincentamen71@gmail.com

ORCID: 0000-0001-9907-8307

Department of Computer Science \& Information Technology

Igbinedion University

Okada

NIGERIA

Virtue U. Ekhosuehi

email: virtue.ekhosuehi@uniben.edu

ORCID: 0000-0002-7796-1657

Department of Statistics

University of Benin 
Benin City

NIGERIA

Augustine A. Osagiede

email: augustine.osagiede@uniben.edu

ORCID: 0000-0002-6835-7925

Department of Mathematics

University of Benin

Benin City

NIGERIA

Received 02.09.2019

Accepted 21.01.2020 